\title{
In vitro efficacy of an anti-microbial solution in prevention of micro-flora colonization of denture-base materials
}

Carneiro LC ${ }^{1}$, Botha $\mathrm{SJ}^{2}$, Kemp PL ${ }^{3}$

${ }^{1}$ Department of Restorative Dentistry, School of Dentistry, Muhimbili University College of Health Sciences, Dar es salaam, Tanzania.

${ }^{2}$ Centre for Stomatological Research, School of Dentistry, University of Pretoria, Pretoria, South Africa.

${ }^{3}$ Department of Prosthetic Dentistry, School of Dentistry, University of Pretoria, Pretoria, South Africa.

Carneiro LC, Botha SJ, Kemp PL ' In vitro Efficacy of an Anti-microbial Solution in Prevention of Micro-flora Colonization of Denture-base Materials : Tanz Dent J. 2007; 14 (1): 15-25

\begin{abstract}
Aim: To evaluate the in vitro efficacy of a new biologically acceptable Anti Microbial-Solution (AMS) for prevention of colonization of polymeric denture base materials. Materials and methods: Sample discs of $6.25 \mathrm{~mm}$ in diameter and $2 \mathrm{~mm}$ thick were sectioned from molded rods using two types of Poly Methylmethacrylate resins (heat and chemical polymerizing). Rate of absorption for the different sample discs was determined for water and for AMS. The concentrations of AMS effective against Streptococcus mutans, Lactobacillus paracasei, Actinomyces naeslundii, Prevotella intermedia, Enterococcus faecalis and Candida albicans were determined and scanning electron microscopy (SEM) was used to confirm colonization of samples. Antimicrobial efficacy of absorbed AMS in the resins was also evaluated. Results: The absorption rate of AMS and water was the same and maximum absorption of about $1 \%$ was reached after $48 \mathrm{~h}$ of soaking. SEM confirmed that the organisms were able to colonize the material surfaces regardless of type of material. AMS was more effective against anaerobic organisms and less effective against aerobic organisms and inhibition of growth of organisms by AMS started at $10 \%$ concentration. Although inhibition was observed with absorbed AMS, no consistent inhibition pattern was found. Conclusion: Efficacy of AMS in the prevention of micro-flora colonization of denture-base materials is reported and AMS is shown to be more effective against anaerobic than aerobic organisms. Lack of optimization of the absorption coefficient of the polymeric resin and the determination of effective concentration of AMS may have led to inconsistent inhibition patterns.
\end{abstract}

Key Words: In vitro, Prevention, Micro-flora Colonization, Denture-base Materials

Correspondence: Dr Lorna C Carneiro, P. O. Box 65451, Dar es salaam, Tanzania, Email: 1carneiro@muchs.ac.tz

Re-submission Date: $30^{\text {th }}$ May 2007

\begin{abstract}
Introduction
Patients using polymeric denture base materials as implant and other prosthesis are faced with the problem of microorganisms colonizing their prostheses irrespective of the various hygiene procedures adopted (1). The oral microbes comprise a complex community, and oral health or disease depends on the interface between the host and the microbial community as a whole (2). The intimate presence of microorganisms in the oral mucosa may elicit adverse reactions that are known to be detrimental to these tissues (3). Any prosthesis must meet the patient's requirements in terms of function and appearance, whilst contributing to the long-term stability of the prosthesis host interface on which the ultimate success of the treatment depends. Three factors are important in maintenance of the interface: plaque control, gingival integrity and interfacial force transmission (4). Like the natural tooth surface, a prosthesis provides a fluid-conditioned surface for colonization of microbial bio-films (5). The denture
\end{abstract}

pellicle, which consists of salivary and serum proteins, facilitates adherence and colonization (plaque formation) on the prosthesis $(6,7)$ and represents a protective reservoir for oral microbes (8). Micro-flora has been reported to colonize and penetrate denture-base materials easily (9) and irregularities of $1-12 \mu \mathrm{m}$ in depth on the surface of Poly-Methylmethacrylate (PMMA) have been recorded (10). This implies that colonization by microorganisms such as cocci with diameters of \pm $1 \mu \mathrm{m}$ and fungi with diameters of $\pm 5 \mu \mathrm{m}$ would be difficult to dislodge from these indentations by mechanical action such as brushing $(11,12)$.

Although numerous types of denture cleaners have been commercialized (1, 3, 13-17), scant information is available concerning their bio-acceptability. This is probably due to employment of these preparations on dentures outside the oral cavity. Most denture cleansers have their common purpose directed 
towards elimination of existing colonized micro-flora (18) and not at prevention thereof.

The aim of this study was to employ an in vitro model to assess the efficacy of a new biologically acceptable anti-microbial solution (AMS) $)_{1}$ for the prevention of micro-flora colonization on polymeric denture-base materials.

\section{Materials and methods}

\section{Preparation of sample discs}

Rods with dimensions of 50 X $6.25 \mathrm{~mm}$ were molded using two Poly-Methylmethacrylate resins: a heat polymerizing (Vertex simplified Rapid) (HC) and a chemical polymerizing (Vertex Castapress) (CC) denture base materials (Dentimax B.V, Xeist, Netherlands). The powder-liquid ratios of the heatcuring and chemical-curing counterpart were $30 \mathrm{~g}: 10 \mathrm{ml}$ and $9.5 \mathrm{~g}: 10 \mathrm{ml}$ respectively. The heat-cured rods were processed in boiling water for $40 \mathrm{~min}$. Processing of the chemical-curing material was accomplished in a pneumatic curing unit (Polymax 3, Dreve, Unna, Germany) under pressure of $200 \mathrm{kPa}$ at $40^{\circ} \mathrm{C}$ water temperature and a curing time of $15 \mathrm{~min}$. The rods were sectioned into discs of $6.25 \times 2 \mathrm{~mm}$ (Fig I) by use of an ISOMET $^{\mathrm{TM}}$ low speed cutting saw (Buehler, Lake Bluff, Illinois, USA) and all surfaces were then hand finished by wet grinding with 600 grit $\mathrm{SiC}$ paper.

\section{Determination of the rate of absorption}

Sample discs were measured using a vernier caliper to ensure dimensions of $6.25 \mathrm{X} 2 \mathrm{~mm}$. Discs were weighed using a Precisa 205A SuperBal-series, Swiss Quality microgram scale prior to immersing in $50 \%$ and $100 \%$ AMS $(\mathrm{n}=8$ (4HC and 4CC)) and distilled water ( $\mathrm{n}=4$ (2HC and 2CC)) for a time interval of 24 , 48, 72h and 1 week. Each disc was placed in one $\mathrm{ml}$ of the different liquids within a capped test-tube and soaking took place in a shake incubator at $37^{\circ} \mathrm{C}$. The discs were weighed repeatedly at every time interval after removing the excess liquid using sterile filter paper. Absorption was determined as weight gain of the resin as function of initial weight of the unsoaked sample discs $(19,20)$.

Rate of Absorption $=$ Weight after soaking $(\mathrm{g})-$ Weight before soaking (g) X 100\%

Weight before soaking $(\mathrm{g})$

Preparation of microorganisms

${ }^{1}$ The AMS liquid that has been used in this study is of an ionic makeup and is under patent; therefore the active ingredients cannot be made known. It has a proven anti-microbial activity at $100 \%$ concentration.
Amongst the bacteria that habour the oral cavity only few bacteria were chosen as representative organisms of commonly known oral diseases namely Streptococcus mutans, Lactobacillus paracasei, Actinomyces naeslundii, Enterococcus faecalis and Candida albicans. McFarland Standard I suspensions (21) of the different test organisms were prepared using a spectrophotometer. Blood agar and Sabouraud Dextrose agar plates were inoculated with $0.1 \mathrm{ml}$ of McFarland Standard Suspensions of Streptococcus mutans, Actinomyces naeslundii, Lactobacillus paracasei, Enterococcus faecalis and Candida albicans respectively. Even spreading of the inoculants on the agar plates was done using a sterile glass spreader (22). All plates were placed in an incubator at $37^{\circ} \mathrm{C}$ for $10 \mathrm{~min}$ to dry prior to placing of test samples. Anaerobiasis was achieved by the use of Anaroucult A.

Determining the effective concentration of AMS

Different dilutions ranging from $100 \%$ down to $0.1 \%$ concentrations of the AMS liquid were prepared using sterile distilled water; $0.5 \mathrm{mls}$ of the different dilutions was measured onto a sterile filter paper $(12 \mathrm{~mm}$ diameter) and placed in duplicate on the spread plates with test organisms (Fig II). All plates were incubated at $37^{\circ} \mathrm{C}$ in respective aerobic (Candida albicans, Streptococcus mutans) and anaerobic (Actinomyces naeslundii, Lactobacillus paracasei, Enterococcus faecalis) atmosphere up to $72 \mathrm{~h}$. The formation of a halo bigger than $0.5 \mathrm{~mm}$ was assumed to indicate antimicrobial activity of AMS, and formed halos were measured in four different areas with a vernier caliper after 24,48 and $72 \mathrm{~h}$ (23).

\section{Antimicrobial efficacy of absorbed AMS}

Soaking of heat polymerizing and chemical polymerizing Poly Methylmethacrylate resins sample discs was done for 24, 48 and $72 \mathrm{hrs}$ in $1 \mathrm{ml}$ of $100 \%$ AMS and 50\% AMS concentrations. Discs were soaked in a shake incubator at $37^{\circ} \mathrm{C}$ for the different time intervals after which they were placed in duplicate on spread plates of the different test organisms (Fig III). The anti-microbial efficacy of the absorbed AMS in the samples was then quantified by measuring the zone of inhibition that surrounds the resin sample with a vernier caliper. 
Electron microscopy of colonization of polymeric denture-base materials

A series of sample discs of the heat polymerizing and chemical polymerizing Poly-Methylmetacrylate resins were placed on the prepared spread plates of the different organisms and incubated for $48 \mathrm{~h}$. After incubation the sample discs were removed from the spread plates and fixated in $2.5 \%$ Glutaraldehyde in $0.075 \mathrm{M}$ phosphate buffer at a $\mathrm{pH}$ of 7.4-7.6 for one hour. The wells were then rinsed three times each for $5 \mathrm{~min}$ with $0.25 \mathrm{mls}$ of $0.075 \mathrm{M}$ phosphate buffer, treated with $0.25 \%$ aqueous osmium tetra-oxide for half an hour in a fume hood and rinsed again three times each for five min with distilled water in the fume hood. Dehydration of the samples was then done with varying concentrations of ethanol of $30 \%$, $50 \% 70 \% 96 \%, 96 \%$ and $96 \%$ each for $5 \mathrm{~min}$. Each specimen was dried in a critical point dryer before being coated with a layer of gold (24). Using a scanning electron microscope (JEOL SM 840, Jeol, Tokyo) the microscopic structure of the heat cured and chemically cured materials were assessed with and without microflora colonization on the surface. All data observed were stored as image files. Experimental procedures were carried out at the Department of Electron Microscopy, University of Pretoria, South Africa, 2002.
Statistical analysis

Means and SD were determined for sample discs weighed repeatedly at every time interval and the measures of inhibition zones. Analysis by the Paired $\mathrm{T}$ - test was done to compare the mean absorption rate and mean inhibition zones at different time intervals. ANOVA was performed so as to determine the rate of absorption, effective concentration of AMS and antimicrobial efficacy of the absorbed AMS between the different concentrations and times. Statistical significance was determined at a p-value $<0.05$ and program used was Statistix 7, Analytical Software, PO Box 12185, Tallahassee FL 32317-2185, 2000.

\begin{abstract}
Results
Determination of the rate of absorption

Rate of absorption (\%) varied over time for the different samples of standardized thickness and smoothness. The volume of all samples was calibrated to $245.46 \mathrm{~mm}^{3}$ and their Surface area was $100.64 \mathrm{~mm}^{2}$. The uptake of liquid was determined by weight gain over a time interval $(19,20)$. The mean absorption rates for the samples are given in Table 1 . For all the samples that were soaked in the different soaking solutions, statistical analysis by the Paired Ttest showed a significant increase $(\mathrm{p}<0.05)$ in weight from 24 to 48 hours, but subsequent weight gains (from $72 \mathrm{~h}$ to 4 weeks) was not statistically significant $(\mathrm{p}>0.05)$.
\end{abstract}

Table 1. Mean absorption rate (\%) of two Poly-Methylmethacrylate resins: a heat polymerizing (Vertex simplified Rapid) (HC) and a chemical polymerizing (Vertex Castapress) (CC) denture base materials (Dentimax B.V, Xeist, Netherlands) after soaking in AMS (100\% and 50\%) and distilled water for time intervals of $24,48,72 \mathrm{~h}, 1,2,3$ and 4 weeks.

\begin{tabular}{lllllccc}
\hline $\begin{array}{l}\text { Material and Soaking } \\
\text { Solution }\end{array}$ & 24h & $48 \mathrm{~h}$ & $72 \mathrm{~h}$ & $1 \mathrm{wk}$ & $2 \mathrm{wks}$ & $3 \mathrm{wks}$ & $4 \mathrm{wks}$ \\
\hline CC in Water & 0.6420 & 1.0780 & 1.2860 & 1.4560 & 1.3420 & 1.3420 & 1.3140 \\
& \pm 0.357 & \pm 0.316 & \pm 0.342 & \pm 0.526 & \pm 0.376 & \pm 0.273 & \pm 0.236 \\
CC in 50\% AMS & 1.0064 & 1.2943 & 1.3305 & 1.1211 & - & - & - \\
& \pm 0.159 & \pm 0.105 & \pm 0.131 & \pm 0.308 & & & \\
CC in 100\% AMS & 0.6120 & 0.9040 & 1.1960 & 1.1100 & 1.2860 & 1.3140 & 1.0520 \\
& \pm 0.316 & \pm 0.193 & \pm 0.157 & \pm 0.336 & \pm 0.274 & \pm 0.103 & \pm 0.286 \\
HC in Water & 0.5980 & 1.0120 & 0.8880 & 1.0720 & 1.1000 & 1.1940 & 1.0160 \\
& \pm 0.200 & \pm 0.247 & \pm 0.133 & \pm 0.166 & \pm 0.119 & \pm 0.222 & \pm 0.148 \\
& & & & & & & \\
HC in 50\% AMS & 1.0528 & 1.2020 & 1.3907 & 1.1301 & - & - & - \\
& \pm 0.121 & \pm 0.235 & \pm 0.134 & \pm 0.184 & & & \\
HC in 100\% AMS & 0.5400 & 0.8740 & 0.9360 & 0.7254 & 0.9940 & 0.9040 & 0.8460 \\
& \pm 0.312 & \pm 0.270 & \pm 0.327 & \pm 0.273 & \pm 0.211 & \pm 0.309 & \pm 0.167 \\
\hline
\end{tabular}


Determining the effective concentration of AMS

The mean inhibition zones after exposure of the test organisms to different concentrations of AMS on filter paper is given in Table 2. Results showed that a concentration of $10 \%$ AMS had no inhibitory effect on S.mutans, E. feacalis and C. albicans, and $20 \%$ AMS had no inhibitory effect on E. feacalis and $C$. albicans. Each increase in the concentration of AMS showed a statistically significant increase $(\mathrm{p}<0.05)$ in the inhibition zone for all the organisms when compared to the lower concentration's inhibition zone. L. paracasei and A. naeslundii were the most sensitive to AMS as inhibition zones were observed at a concentration of $10 \%$ AMS. Various inhibition zones were observed for the different organisms (Fig IV).

\section{Antimicrobial efficacy of absorbed AMS}

After sample discs were soaked in $100 \%$ AMS and $50 \%$ AMS for $48 \mathrm{~h}$, the mean zones of inhibition that surrounds the different resin samples for the different test organisms are given in Table 3. Resin sample discs soaked in $100 \%$ AMS only exhibited zones of inhibition for Actinomyces naeslundii. Although zones were present, it was not zones of complete inhibition, as a fine growth of the organism was still observed. Fig V shows that for most organisms there was absence of an inhibition zone for the different resin samples.

Table 2: Mean inhibition zones (mm) for the different test organisms after exposure to different oncentrations of AMS on filter paper as measured after 24,48 and $72 \mathrm{~h}$ incubation of agar plates.

\begin{tabular}{|c|c|c|c|}
\hline Organism and AMS Concentration & Zone $-24 \mathrm{~h}$ & Zone $-48 \mathrm{~h}$ & Zone $-72 \mathrm{~h}$ \\
\hline Streptococcus mutans - 10\% AMS & 0 & 0 & 0 \\
\hline Streptococcus mutans - 20\% AMS & $1.32 \pm 0.80$ & $0.61 \pm 0.21$ & $0.62 \pm 0.19$ \\
\hline Streptococcus mutans - 50\% AMS & $4.55 \pm 0.58$ & $4.41 \pm 0.55$ & $4.19 \pm 0.41$ \\
\hline Streptococcus mutans - 100\% AMS & $7.93 \pm 0.72$ & $7.54 \pm 0.65$ & $8.03 \pm 0.65$ \\
\hline Lactobacillus paracasei-10\% AMS & $0.58 \pm 0.20$ & $0.57 \pm 0.18$ & $0.41 \pm 0.11$ \\
\hline Lactobacillus paracasei-20\% AMS & $1.48 \pm 0.42$ & $1.45 \pm 0.46$ & $1.43 \pm 0.53$ \\
\hline Lactobacillus paracasei-50\% AMS & $4.26 \pm 0.49$ & $3.59 \pm 0.63$ & $3.57 \pm 0.80$ \\
\hline Lactobacillus paracasei-100\% AMS & $6.31 \pm 0.52$ & $6.52 \pm 0.75$ & $6.47 \pm 0.67$ \\
\hline Enterococcus faecalis - 10\% AMS & 0 & 0 & 0 \\
\hline Enterococcus faecalis - $20 \%$ AMS & 0 & 0 & 0 \\
\hline Enterococcus faecalis - 50\% AMS & $1.27 \pm 0.39$ & $0.58 \pm 0.26$ & $0.63 \pm 0.17$ \\
\hline Enterococcus faecalis - 100\% AMS & $4.27 \pm 0.49$ & $6.75 \pm 0.46$ & $4.43 \pm 0.58$ \\
\hline Actinomyces naeslundii-10\% AMS & $2.02 \pm 1.63$ & $2.39 \pm 1.12$ & $2.18 \pm 1.32$ \\
\hline Actinomyces naeslundii-20\% AMS & $4.83 \pm 1.48$ & $4.85 \pm 1.16$ & $2.37 \pm 0.49$ \\
\hline Actinomyces naeslundii-50\% AMS & $4.62 \pm 0.60$ & $5.57 \pm 0.76$ & $5.20 \pm 0.73$ \\
\hline Actinomyces naeslundii-100\% AMS & $8.95 \pm 2.77$ & $8.43 \pm 1.79$ & $7.00 \pm 1.66$ \\
\hline Candida albicans $-10 \%$ AMS & 0 & 0 & 0 \\
\hline Candida albicans $-20 \%$ AMS & 0 & 0 & 0 \\
\hline Candida albicans $-50 \%$ AMS & $4.51 \pm 1.07$ & $2.75 \pm 1.54$ & $2.05 \pm 1.41$ \\
\hline Candida albicans $-100 \%$ AMS & $6.15 \pm 0.65$ & $6.56 \pm 0.85$ & $6.51 \pm 0.50$ \\
\hline
\end{tabular}

Electron microscopy of colonization of polymeric denture-base materials

Colonization of the sample discs of the heat polymerizing and chemical polymerizing PolyMethylmethacrylate resins by the different test organisms is shown in Figures VI and VII. Voids were observed adjacent to the polymer beads in chemical polymerized materials unlike the heat polymerized materials which appeared to have a uniform blend with no obvious beads. Cell division, budding and mucin production by the microorganisms on sample discs was also observed 
Table 3: Mean zones of inhibition ( $\mathrm{mm})$ for tested microorganisms after exposure to resin that has been soaked in $100 \%$ and $50 \%$ AMS for 24, 48 and 72 hours respectively.

\begin{tabular}{llll}
\hline Organism and AMS Concentration & $\begin{array}{l}\text { Zone }- \\
24 \mathrm{~h}\end{array}$ & $\begin{array}{l}\text { Zone }- \\
48 \mathrm{~h}\end{array}$ & $\begin{array}{l}\text { Zone } \\
-72 \mathrm{~h}\end{array}$ \\
\hline Streptococcus mutans - 50\% AMS & 0 & 0 & 0 \\
Streptococcus mutans - 100\% AMS & 0 & 0 & 0 \\
Lactobacillus paracase - 50\% AMS & 0 & 0 & 0 \\
Lactobacillus paracasei - 100\% AMS & 0 & 0 & 0 \\
Enterococcus faecalis - 50\% AMS & 0 & 0 & 0 \\
Enterococcus faecalis - 100\% AMS & 0 & 0 & 0 \\
Actinomyces naeslundii-50\% AMS & 0 & 0 & 0 \\
Actinomyces naeslundii- 100\% AMS & 0 & $\pm 5^{*}$ & $\pm 5^{*}$ \\
Candida albicans - 50\% AMS & 0 & 0 & 0 \\
Candida albicans - 100\% AMS & 0 & 0 & 0 \\
\hline
\end{tabular}

Although zones were present, it was not zones of complete inhibition, as a fine growth of the organism was still observed.

\section{Discussion}

The polymers used in prosthetic dentistry are often multiphase acrylic resins made from pre-polymerized powder beads of Poly Methylmethacrylate (PMMA) and a liquid of monomers such as Methylmethacrylate (MMA). These dentures are continuously exposed to oral micro-flora and frequently colonized by high numbers of normal or pathogenic flora of the oral cavity. Roughness and porosity of the acrylic surface may favor initial formation of plaque by protecting the organisms from dislodgment (10). Research has been conducted over the years to establish a way of minimizing colonization of microorganisms on denture base materials.

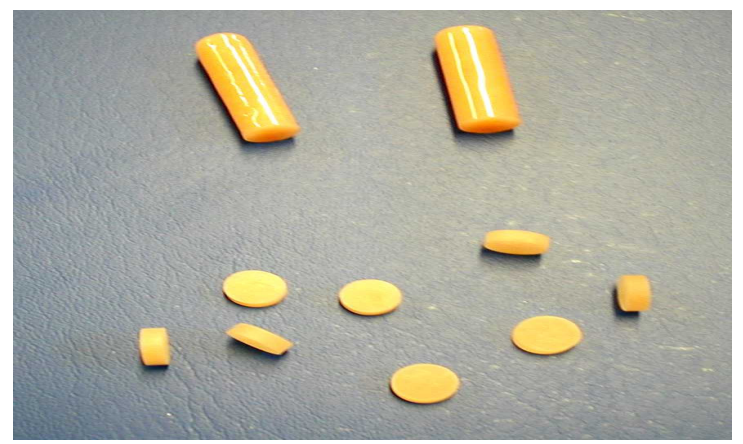

Figure I: Prepared rods of PolyMethylmethacrylate resins were sectioned into discs of $6.25 \times 2 \mathrm{~mm}$.

Currently denture cleansers in use are either pastes or immersion types (15). Traditionally the most common method of removal of denture plaque was brushing with an abrasive paste, whilst chemical soaking agents are the second most popular method of denture cleansing (25). Chemical soaking solutions for cleaning dentures may be denture cleansers or disinfectants. Commercial denture cleansers are classified according to their mode of action or their main component (26). Of the available disinfectants, sodium hypochlorite is one of the earliest and most widely used disinfectants (27) and relatively efficient in their cleansing ability but can lead to deterioration of the denture base material (28). Other attempts against microbial colonization have been the use of viscogel with antifungal/antibiotics (29) but some of the disinfectants and commercial cleaners have been reported to cause deterioration of tissue conditioners and/or lining materials and also mechanical cleansing has been reported to be harmful to soft lining materials (30). With the effective cleansing of dentures in mind, many models have been developed over the years, but to date no treatment has managed to curb the situation and patients are still faced with the problem. This study has laid the basis for future work to develop and optimize the conditions for an effective anti-microbial agent for prevention of microbial colonization.

Although it can be relative difficult to standardize sample size with different dental materials, measurements of the samples confirmed the standardization of sample size as in accordance with the American National Standard/American Dental Association specification No. 12 (31). The preparation of standardized sample discs was important to allow interpretation of results and further fabrication of samples required for follow- up or comparative studies. 


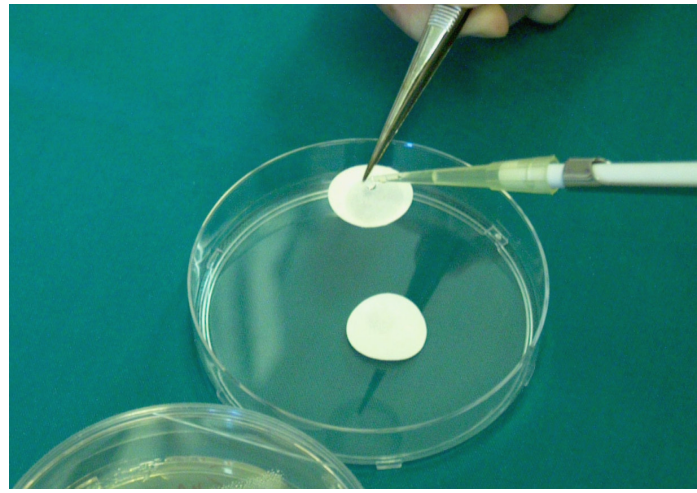

Figure II: The different dilutions of AMS were measured onto a sterile filter paper $(12 \mathrm{~mm}$ diameter) and placed in duplicate on the spread plates with test organisms

Poly Methylmethacrylate (PMMA) absorbs water slowly over a period of time $(19,20)$. In this study, we determined the rate of absorption to be at its maximum after $48 \mathrm{hrs}$ of soaking. Several interpretations of rate of absorption were noted in the literature $(31,32)$.

In this study it was decided to use the rate of absorption that is expressed in percentage (\%) as special care was taken to standardize sample size (31). The rate of absorption varied over time for the different samples. Statistical analysis for all the samples that were soaked in the different soaking solutions showed that there was a significant increase $(\mathrm{p}<0.05)$ in weight from 24 to 48 hours, but subsequent weight gains (from $72 \mathrm{~h}-4$ weeks) were not statistically significant $(p>0.05)$. These results imply that the resins were practically saturated with the different soaking solutions after $48 \mathrm{~h}$ and therefore soaking for longer than $48 \mathrm{~h}$ will not have a significant effect on uptake of AMS.

Mean inhibition zones showed that a concentration of $10 \%$ AMS had no inhibitory effect on S.mutans, E. feacalis and $C$. albicans, and 20\% AMS had no inhibitory effect on E. feacalis and C. albicans. Each increase in the concentration of AMS showed a statistically significant increase $(p<0.05)$ in the inhibition zone for all the organisms when compared to the lower concentration's inhibition zone. The zone of inhibition for $E$. feacalis was relatively small at $50 \%$ AMS, but all the other organisms showed zones larger than $4 \mathrm{~mm}$ after 24 hours, which indicate very good inhibitory properties. It was interesting to note that some organisms (C. albicans at 50\% AMS) had the ability to reduce the inhibition zone after longer incubation, as this may indicate towards the development of resistance and should be investigated properly before the application of AMS as denture soaking solution. This adaptation was however not observed in $100 \%$ AMS and it may only be necessary to optimize the AMS concentration to prevent the development of resistance.

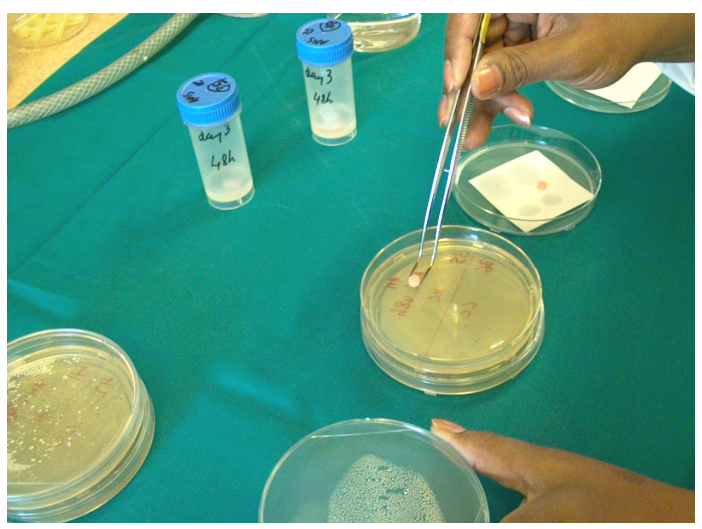

Figure III: Poly-Methylmethacrylate resins sample discs soaked in varying concentrations of AMS were placed in duplicate on spread plates of the different test organisms

L. paracasei and $A$. naeslundii were the most sensitive for inhibition by AMS as inhibition zones were observed at a concentration of $10 \%$ AMS. As $L$. paracase $i$ is a facultative anaerobic organism and $A$. naeslundii is an anaerobic organism both these organisms need a restriction in the oxygen concentration for growth. It may therefore be purely accidental or an intrinsic property of AMS to have a stronger affect on anaerobic organisms. More research needs to be done to confirm this property of AMS.

Inhibition zones observed after soaking resin sample discs in $100 \%$ AMS and 50\% AMS for $48 \mathrm{~h}$ and exposing them then to the different test organisms indicated that only A. naeslundii was inhibited by $100 \%$ AMS and this inhibition was not complete. It is possible that due to the small disc size a smaller amount of AMS was absorbed hence not able to withstand the colonization. The results indicate that this type of inhibition may be possible, but the absorption and desorption rate of the resin have to be optimized to ensure that enough of the AMS is absorbed and released to inhibit growth of the microorganisms. 

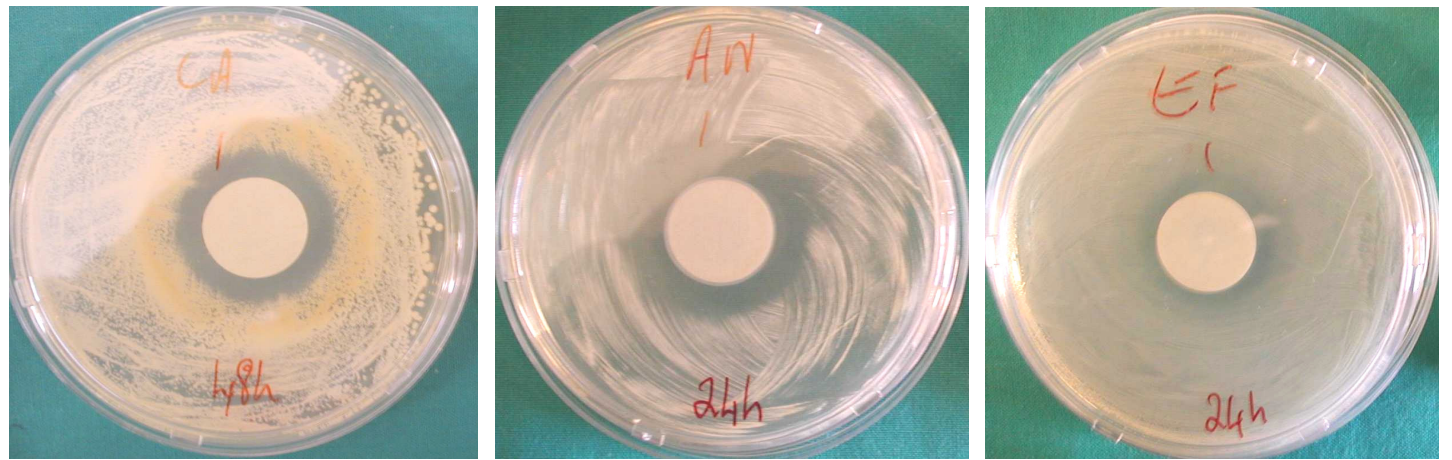

Figure IV: Different concentrations of AMS on filter paper showed varying inhibition zones for the test organisms.

SEM images confirmed the colonization of the CC and $\mathrm{HC}$ resins by all the different test organisms. It was also observed by SEM that all the rough surface areas and air bubbles created during polymerization enhances microbial colonization and actually forms protective niches where bacteria colonize and grow with relative ease (Fig. VI \& VII).

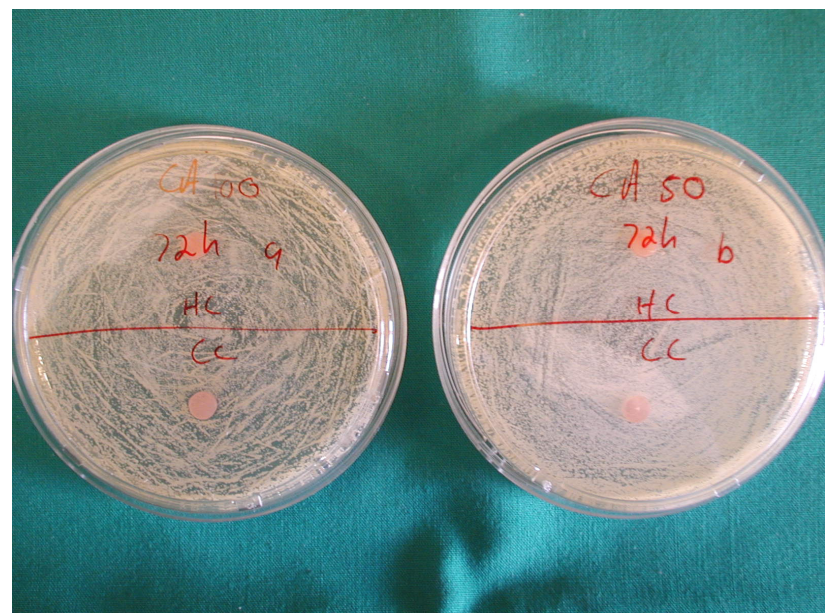

Figure V: Poly-Methylmethacrylate resin discs of either material soaked in different concentrations of AMS displaying no inhibition zones against the test organisms.

The biological features of denture base resins are highly influenced by its monomer-polymer conversion. Despite the various methods to initiate the polymerization of denture base resins, the conversion of monomers to polymers is not complete and some un-reacted monomers are left in the denture base polymers (33). On some images (Fig. VI \& VII) of the CC resin it was evident that this material is more porous than $\mathrm{HC}$ resin and some un-polymerized beads were embedded in the $\mathrm{CC}$ resin. These irregularities created even more surfaces/niches where microorganisms could colonize the resin.

These defects may also make it difficult to remove plaque completely (34). Although mechanical attachment of microbial plaque may not occur on the surface of a denture under normal circumstances, unpolished surfaces of some resins appear to be porous and microorganisms probably penetrate into the superficial layers. Their presence within the impression surface of the denture would make it difficult to remove them even with disinfectants and would provide a niche for infection (10).

Candida albicans are capable of growing on denture surfaces and also capable of growing into the voids/porosities created during fabrication from where they can infect and re-infect soft tissue. Saliva and serum pellicles facilitate multilayered Candida colonization and bio-film formation that is comprised not only of blastospores but also of hyphal emergence (6). Of greater significance than the potential for attachment to PMMA is the question whether microflora are able to penetrate into the denture-base material and thus become totally protected from the external cleaning technique. It has been suggested that fungi can penetrate into the porosity of PMMA and survive by diffusion of essential nutrients through the acrylic resin (10). Interestingly, clinical trials comparing the anti-microbial capacity of various denture cleaners revealed that none of the agents were able to significantly reduce levels of colonizing fungi (25). SEM images of this study show cell division, budding and mucin production that the organisms use for proliferation and attachment to the PMMA surfaces. This produced mucin is possibly what penetrates the voids and allows micro-flora to thrive. 


\section{Conclusions}

The efficacy of AMS in the prevention of micro-flora colonization of denture-base materials is reported and AMS is shown to be more effective against anaerobic than aerobic organisms. Both types of polymeric resin
(CC and $\mathrm{HC}$ ) can absorb AMS to some extent; however, the observed inhibition patterns were not consistent. There is further need to optimize the absorption coefficient of the polymeric resin and the effective concentration of AMS.

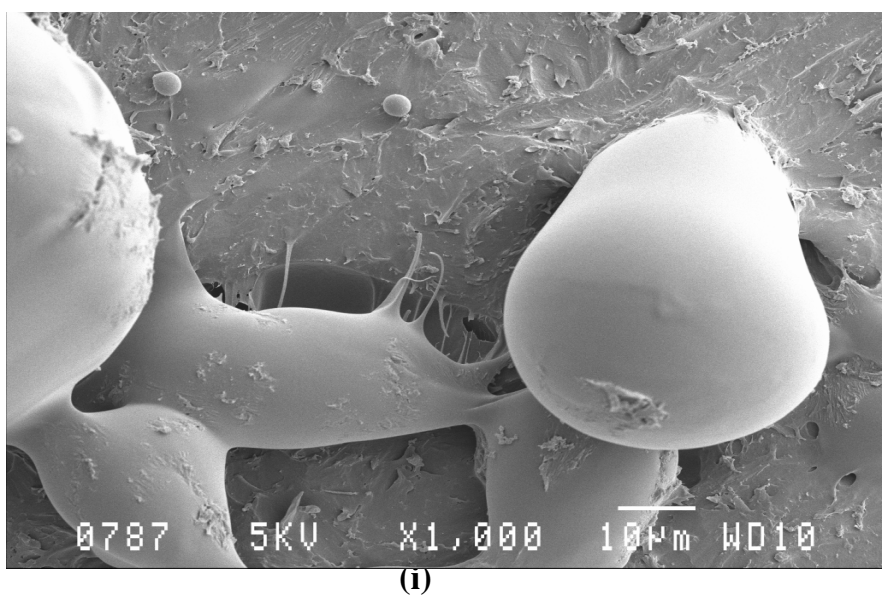

(ii)

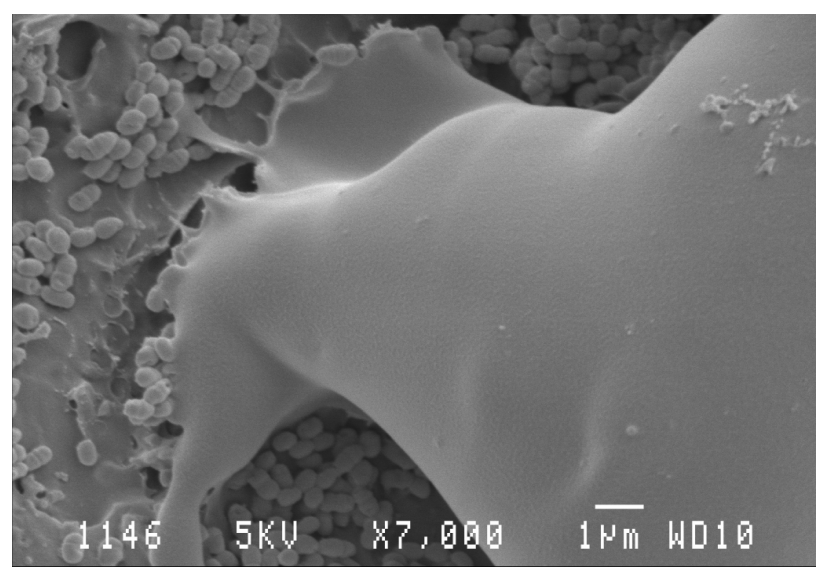

(iv)

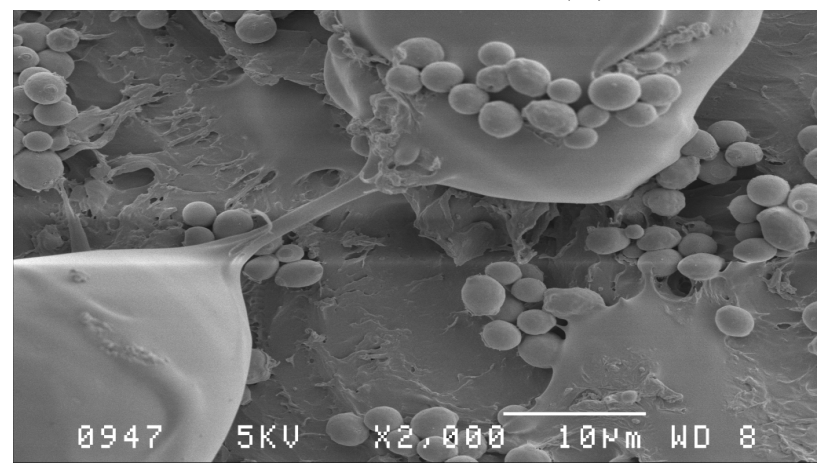

(iii)

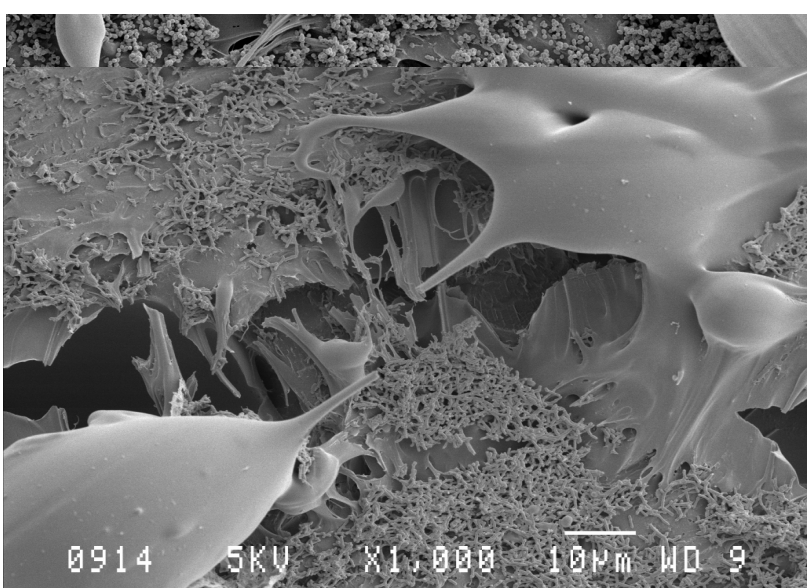

(v)

Figure VI: Scanning Electron Microscopy images of microorganisms colonization on the chemical curing PolyMethylmethacrylate resins (Vertex Castapress = CC). (i) CC Resin without microorganisms, (ii) S.mutans on CC resin (iii) E. feacalis on CC resin (iv) C. albicans on CC resin (v) A. naeslundii on CC resin 


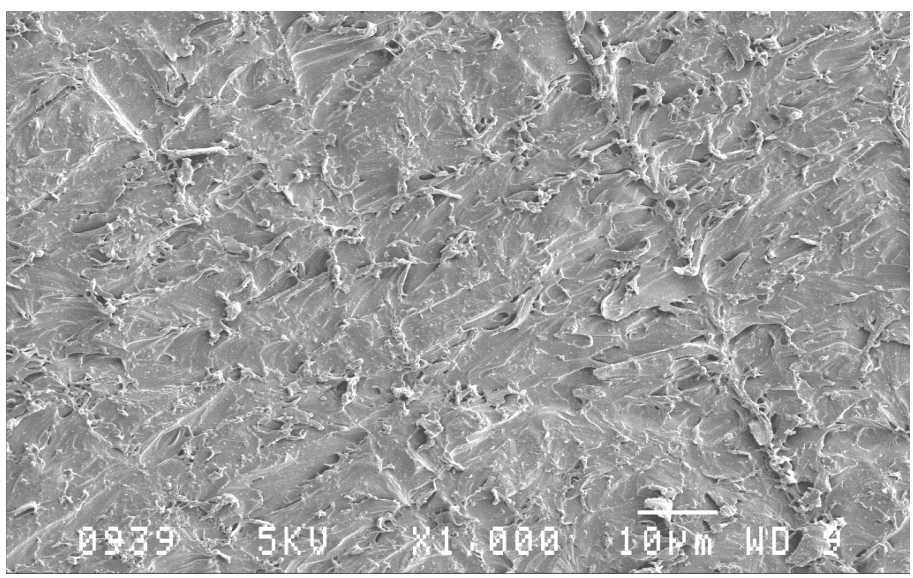

(i)

(ii)

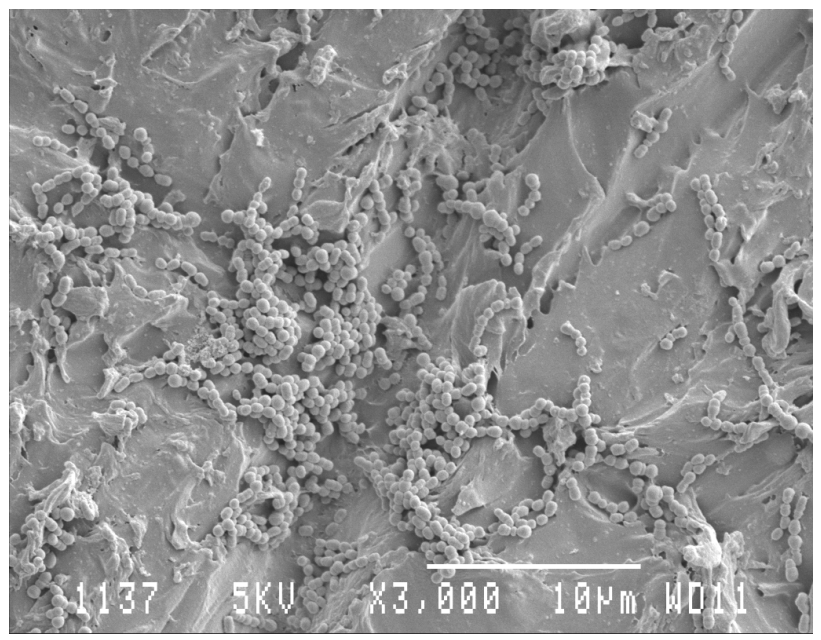

(iv)

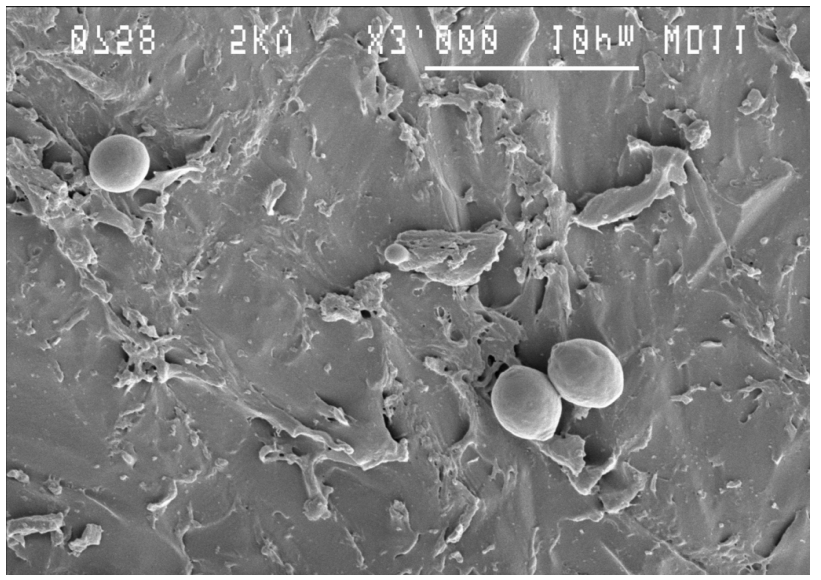

(iii)

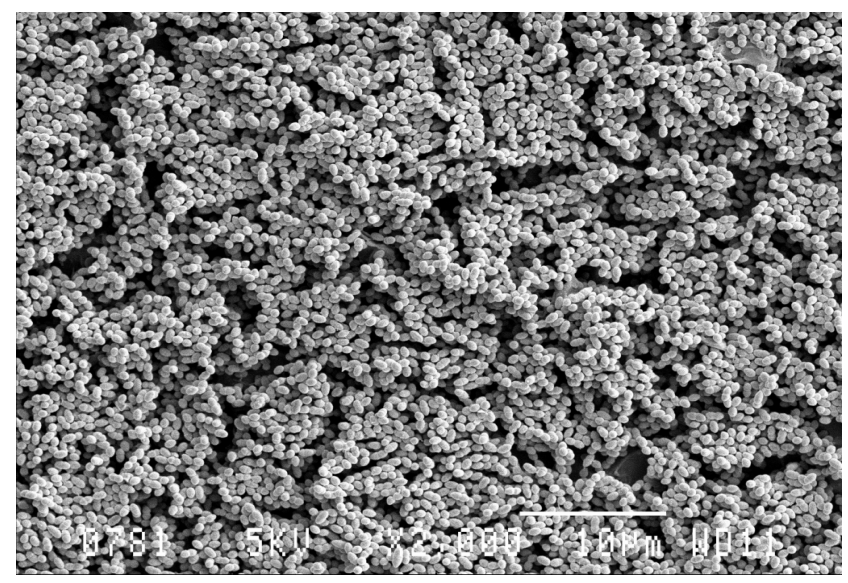

(v)

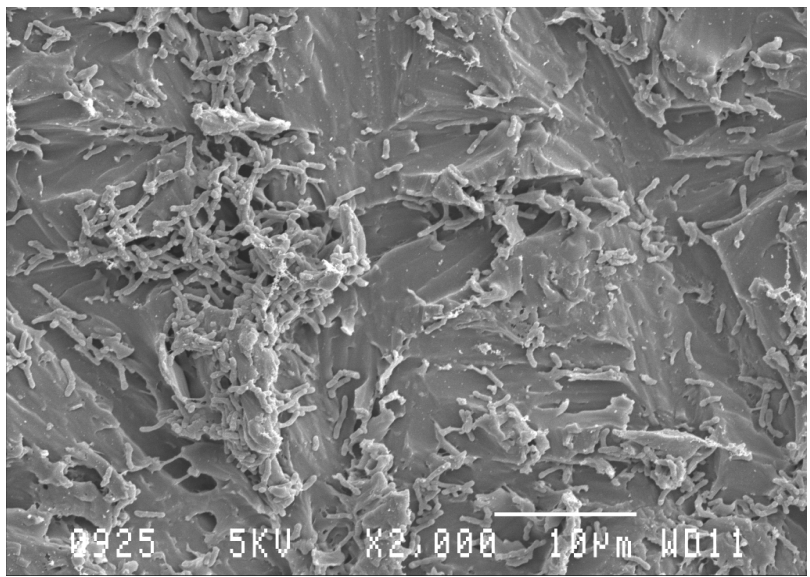

Figure VII: Scanning Electron Microscopy images of microorganisms colonization on the heat polymerizing Poly Methylmethacrylate resins (Vertex Simplified Rapid = HC). (i) HC Resin without microorganisms, (ii) S.mutans on $\mathrm{HC}$ resin (iii) E. feacalis on $\mathrm{HC}$ resin (iv) C. albicans on $\mathrm{HC}$ resin (v) A. naeslundii on $\mathrm{HC}$ resin. 


\section{Acknowledgement}

The authors would like to acknowledge: the manufacturer and supplier of the experimental Antimicrobial Solution (AMS), MAC, P.O. Box 14203, Farrarmere, Benoni, South Africa, 1518, for allowing us to be a platform for evaluating and developing products suitable for human health care; the staff from the departments of Stomatological Research, Prosthetic Dentistry, and Electron Microscopy, University of Pretoria for rendering their assistance in one way or another

\section{References}

1. Shay K: Denture hygiene: a review and update. J Contemp Dent Pract. 2000,15:28-41.

2. Jenkinson HF, Lamont RJ: Oral microbial communities in sickness and in health. Trends Microbiol. 2005,13:589-95.

3. Nikawa $\mathrm{H}$, Jin $\mathrm{C}$, Makihira $\mathrm{S}$, Egusa $\mathrm{H}$, Hamada T, Kumagai H: Biofilm formation of Candida albicans on the surfaces of deteriorated soft denture lining materials caused by denture cleansers in vitro. J Oral Rehabil. 2003,30:243-50.

4. Cannon RD, Chaffin WL: Colonization is a crucial factor in oral candidiasis. Journal of Dental Education. 2001,65:785-787.

5. Ellen RP: Microbial colonization of the periimplant enviroment and its relevance to longterm success of osseointergrated implants. The International Journal of Prosthodontics. 1998,11:433-441.

6. Nikawa H, Havashi S, Nikawa Y, Hamada T, Samaranayake LP: Interactions between denture lining material, protein pellicles and Candida albicans. Archives of Oral Biology. 1993,38:631-634.

7. Jones GW, Isaacson RE: Proteinaceous bacterial adhesions and their receptors. $C R C$ Critical Reviews in Microbiology. 1983,10:229-260.

8. Chandra J, Mukherjee PK, Leidich SD, Faddoul FF, Hoyer LL, Douglas LJ, Ghannoum MA: Antifungal resistance of candidal biofilms formed on denture acrylic in vitro. J Dent Res. 2001,80(3):903-8.

9. Allison RT, Douglas WH: Microcolonization of the denture-fitting surface by Candida albicans. Journal of Dentistry. 1973,1:198201.

10. Van Reenen JF: Microbial studies on denture stomatitis. Journal of Prosthetic Dentistry. 1973,30:493.

11. Kulak Y, Arikan A, Salbak S, Okar I, Kazazoglu E: Scanning electron microscopic examination of different cleaners: Surface contaminant removal from dentures. J Oral Rehabil. 1997,24:209-215.
12. Webb BC, Thomas CJ, Willcox MD, Harty DW, Knox KW: Candida-associated denture stomatitis. Aetiology and management: a review. Part 2. Oral diseases caused by Candida species. Aust Dent J. 1998,43:160-6.

13. Arita M, Nagayoshi M, Fukuizumi T, Okinaga T, Masumi S, Morikawa M, Kakinoki Y, Nishihara T: Microbicidal efficacy of ozonated water against Candida albicans adhering to acrylic denture plates. Oral Microbiol Immunol. 2005,20:206-10.

14. Yilmaz H, Aydin C, Bal BT, Ozcelik B: Effects of disinfectants on resilient denturelining materials contaminated with Staphylococcus aureus, Streptococcus sobrinus, and Candida albicans. Quintessence Int. 2005,36:373-81.

15. Harrison Z, Johnson A, Douglas CW: An in vitro study into the effect of a limited range of denture cleaners on surface roughness and removal of Candida albicans from conventional heat-cured acrylic resin denture base material. J Oral Rehabil. 2004,31:460-7.

16. Goodson LB, Glass RT, Bullard JW, Conrad RS: A statistical comparison of denture sanitation using a commercially available denture cleaner with and without microwaving. Gen Dent. 2003,51:148-51.

17. Jagger DC, Al-Akhazam L, Harrison A, Rees JS: The effectiveness of seven denture cleansers on tea stain removal from PMMA acrylic resin. Int J Prosthodont. 2002,15:54952.

18. Nikawa N, Yamamoto T, Hamada T, Sadamori S, Agrawal S: Cleansing efficacy of commercial denture cleaners: Ability to reduce Candida albicans biofilm activity. The International Journal of Prosthodontics. 1995,8:527-534.

19. Braden M: The absorption of water by acrylic resins and other materials. Journal of Prosthetic Dentistry. 1964,14:307-316.

20. Miettinen VM, Vallittu PK: Water sorption and solubility of glass fibre-reinforced denture polymethylmetacrylate resin. Journal of Prosthetic Dentistry. 1997,77:531-4.

21. MacFarland J. The nephelometer: an instrument for estimating the number of bacteria in suspensions for calculating the opsonic index and for vaccines. $J$ Am Med Assoc 1907; 49:1176

22. Gerhardt P, Murray RGE, Costilow RN, Nester EW, Wood WA, Krieg RN \& Phillips GB: Manual of methods for general bacteriology. Washington: American Society for Microbiology, 1981;524pp.

23. Johnson $\mathrm{T}$ and Case C. Laboratory Experiments in Microbiology, Brief Edition, 
4th ed., Redwood City, CA: Benjamin/Cummings Publishing Co., 1995

24. Bullock GR \& Petrusz P (1985) Techniques in Immunochemistry. London: Academic Press.

25. Dills SS, Olshan AM, Goldner S, Brogdon C: Comparison of the anti-microbial capability of an abrasive paste and chemical-soak denture cleaners. Journal of Prosthetic Dentistry. 1988,60:467-470.

26. Budtz-Jorgensen E: Materials and methods for cleaning dentures. Journal of Prosthetic Dentistry. 1979,42:619-623

27. Dychdala GR: Disinfection, Sterilization and preservation. $5^{\text {th }}$ edn. Philadelphia:Lea and Febiger; 1991:133

28. Harrison A, Jagger DC: An in vitro investigation of the abrasive qualities of a selection of denture cleaning pastes on poly (methyl methaacrylate) denture base materials. Prim Dent Care. 1997,4:21-4.

29. Lacopino AM, Wathen WF: Oral Candidal infection and denture stomatitis: a comprehensive review. Journal of the American Dental Association. 1992,123:46-51.
30. Nikawa H, Iwanaga H, Ganefivanti $T$, Hamada T: Effects of denture cleansers on soft lining materials. Journal of Prosthetic Dentistry. 1994,72:657-662.

31. ANSI/ADA: American National Standard / American Dental Association Specification no. 12. Denture Base Polymers. Chicago. 1999;p8

32. International Organization for Standardization, Specification 1567: Dentistry-denture base polymers. $2^{\text {nd }}$ ed., Geneva Switzerland: International Standards Organization. 1988.

33. Huang F-M, Tai K-W, Hu C-C, Chang Y-C: Cytotoxic effects of denture base materials on a permanent human oral epithelial cell line and on primary human oral fibroblasts in vitro. The International Journal of Prosthodontics. 2001,14:439-443.

34. Waltimo T, Vallittu P, Haapasalo M: Adherence of Candida species to newly polymerized and water-stored denture base polymers. The International Journal of Prosthodontics. 2001,14: 457-460. 